\title{
MiReBooks - Mixed Reality Lehrbücher für das Bergbau-Studium
}

\author{
Hanno Bertignoll, Manuel Labrador Ortega, Susanne Feiel und Peter Moser \\ Resources Innovation Center RIC Leoben, Montanuniversität Leoben, Leoben, Österreich \\ Eingegangen 5. Februar 2019; angenommen 6. Februar 2019; online publiziert 20. Februar 2019
}

Zusammenfassung: Die Montanuniversität Leoben leitet das europaweite Projekt MiReBooks des EIT RawMaterials, das die Erstellung neuer Bergbaulehrbücher zum Ziel hat. Mixed Reality Books, kurz MiReBooks, steht für interaktive Lehrbücher, die durch die Integration von digitalen Visualisierungselementen, wie z. B. Virtual und Augmented Reality, die Ausbildung im Bereich Bergbau modernisieren sollen. Ziel ist es, zukünftig in ganz Europa Lehr- und Lernmaterialien für das Bergbaustudium zur Verfügung zu haben, die als neue internationale Standards die traditionelle Wissensvermittlung um erfahr- und erlebbare Komponenten erweitern. Der Ansatz dient auch als Modell für weitere Fachrichtungen.

Schlüsselwörter: Rohstoffsektor, Hochschulbildung, Internationalisierung, Lehre, Digitalisierung, Augmented Reality, Bildungsbedarf, EIT, Innovation, Bewusstseinsbildung, Rohstoffe, Ressourcen, Nachhaltigkeit, Weiterbildung, Wissenschaft, Industrie, Forschung

\section{MiReBooks-Mixed Reality Handbooks for Mining Education}

Abstract: Montanuniversitaet Leoben is leading a pan-European project to create new media for mining education. In the project "MiReBooks" a series of virtual and augmented reality-based (=Mixed Reality MR) interactive mining handbooks will be produced as a new digital standard for higher mining education throughout Europe. Many current challenges in mining education will be met in an innovative way, by combining classical paper-based teaching materials with MR materials and their transformation into pedagogically and didactically coherent MR handbooks for

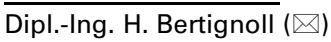

Resources Innovation Center RIC Leoben,

Montanuniversität Leoben,

Franz-Josef-Straße 18,

8700 Leoben, Österreich

hanno.bertignoll@unileoben.ac.at integrative classroom use. The approach will be used in the future also in other disciplines.

Keywords: Raw Materials, Higher Education, Internationalization, Teaching, Digitization, Augmented Reality, EIT, Innovation, Resources, Sustainability, Science, Industry, Research

\section{Der digitale Wandel des Bergbaustudiums in Europa}

Die Herausforderungen im Bergbaustudium sind vielfältig. Dabei bilden besonders die Übertragbarkeit der theoretischen Wissensinhalte in die praktische Tätigkeit und somit auch ein fließender Übergang vom Studium in das Berufsleben eine zunehmend größere Herausforderung. Die „Verfügbarkeit von Bergwerken“ als Orte des Lernens ist nicht nur geografisch sehr beschränkt, sondern zunehmend wird die Zugänglichkeit auch durch immer strenger werdende Sicherheitsvorschriften limitiert und somit auch das praxisnahe Erleben der Studieninhalte. Oft kann die praktische Erfahrung durch die Studierenden nur in den unterrichtsfreien Zeiten, z. B. Sommerferien, gesammelt werden. Dies hat zur Folge, dass nicht alle Studierenden systematisch vergleichbare Erfahrungen sammeln können. Hinzu kommt, dass Literatur aus vergangenen Jahrzehnten immer noch als Grundlagenwerke und Lehrmaterialien genutzt werden, während digitalisierte Materialien kaum zur Verfügung stehen.

Die Montanuniversität Leoben hat daher im Rahmen eines vom EIT RawMaterials geförderten Projekts "MiReBooks - Mixed Reality Handbooks for Mining Education“ die Erstellung modernster Unterrichts-Medien zur Ausbildung im Bereich Bergbau gestartet.

MiReBooks steht für interaktive Lehrbücher, die durch die Integration von digitalen Visualisierungselementen, wie z.B. Virtual und Augmented Reality (VR bzw. AR), die Hochschulbildung im Bereich Bergbau weiter entwickeln sollen. Ziel ist es, zukünftig in ganz Europa Lehr- und Lernmaterialien für das Bergbaustudium zur Verfügung 
Abb. 1: VR-Exkursion zum Steirischen Erzberg

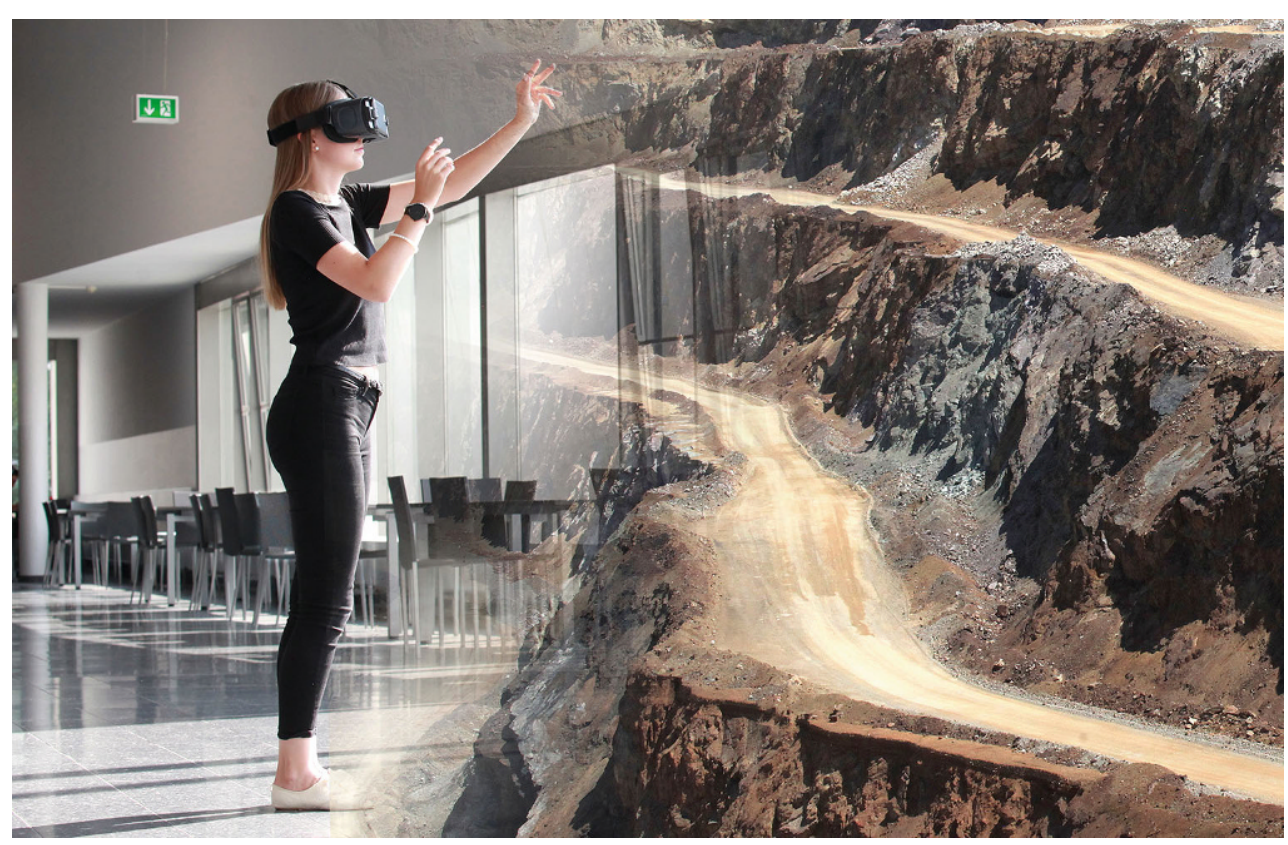

zu stellen, die als neue internationale Standards die traditionelle Wissensvermittlung um erfahr- und erlebbare Komponenten erweitern.

MiReBooks verändert als digitale Lernerfahrung die Art und Weise, wie gelehrt, gelernt und Wissen erfahrbar gemacht wird: Klassische papierbasierte Lehrmaterialien werden mit Mixed Reality (MR)-Inhalten kombiniert und in pädagogisch und didaktisch aufbereitete MR-Handbücher für die interaktive Nutzung im Unterricht umgesetzt. Dadurch können Lernende im Hörsaal Szenarien erleben, die in der Regel schwer oder aufgrund riskanter Sicherheitsbedingungen normalerweise überhaupt nicht zugänglich sind.

Die Möglichkeit, sich frei in einem virtuellen Bergwerk zu bewegen, gehört dabei genauso zu einem zukünftigen Lernszenario wie auch die Betrachtung von Bauteilen oder Maschinen mit Hilfe von Augmented Reality (Abb. 1).

\section{Hintergrund des Projektes}

Die Ausbildung im Rahmen des Bergbaus beinhaltet zahlreiche Bereiche, in denen komplexe dreidimensionale Strukturen und Prozesse vermittelt werden müssen. Hinzu kommt in vielen Fällen eine zeitliche Veränderung der dreidimensionalen Bergbauwelt: Abbauprozesse oder gebirgsmechanische Bewegungen verändern die Position einzelner Elemente über einen Zeitraum. All dies kann derzeit nur über grafische Darstellungen und 3D Animationen geschehen. Räumliches Erleben ist und war schon immer eine wesentliche Komponente der Lernerfahrung und wird sie auch zukünftig sein. Die Studenten müssen dabei jedoch die Fähigkeit besitzen, diese Inhalte in die dreidimensionale Wirklichkeit zu übersetzen. Dabei stoßen sie oft auf Schwierigkeiten. Auch hat sich die Art und Weise, wie sich Studierende heutzutage Wissen aneignen, drama- tisch von analogem Wahrnehmen zum Lernen der Digital Natives verändert. Die heutige Studierendengeneration findet es immer schwieriger, sich Wissen aus traditionellen Druckmedien bzw. im Rahmen eines Frontalunterrichtes anzueignen.

Um den Studenten die für ihre zukünftige Tätigkeit nötige praktische und räumliche Erfahrung erlebbar zu machen, stellten Exkursionen zu Bergbaubetrieben bis dato einen wesentlichen Bestandteil der Ausbildung dar. Der Bergbau ist jedoch ein Industriezweig mit besonderen Herausforderungen, weshalb Betriebsbesuche aus verschiedenen Gründen zunehmend schwieriger zu organisieren sind. Eine ebenso große Herausforderung stellt es oft für die Lehrenden dar, eine Befahrung eines Bergbaubetriebes mit 30 bis 40 Studenten durchzuführen. Dies beginnt bereits bei den vorbereitenden Tätigkeiten, wie z. B. den vorgeschriebenen Sicherheitstrainings für die Besucher. Diese sind bei vielen Betrieben derart komplex, dass ein eintägiger Besuch nicht mehr durchführbar ist. Dadurch wird die Zahl der mit vertretbarem Aufwand befahrbaren Betriebe stets geringer. Eine weitere Schwierigkeit stellt die geografische Lage vieler Betriebe dar. Geeignete Betriebe sind meist nicht in der Nähe der Universitäten zu finden und sind daher nur mit hohem logistischem Aufwand zu erreichen. Das bedeutet für die interessierten Universitäten hohe Kosten bei gleichzeitiger Knappheit der zur Verfügung stehenden Zeit. Für viele Studenten ist es deshalb oft nicht mehr möglich, die nötige praktische Erfahrung vor Ort zu sammeln.

Virtual und Augmented Reality bieten Lösungen und Chancen, diese Barrieren zu überwinden, gerade im Ausbildungssektor. Mixed Reality ist eine wunderbare Möglichkeit, Studierende in eine dynamische und realitätsnahe Umgebung zu transportieren, ohne den Hörsaal verlassen zu müssen. Die erreichten Ergebnisse übertreffen oftmals sogar die bei einem realen Bergbaubesuch gewonnenen Eindrücke. Damit können die Studenten unterstützt wer- 
den, in „realen“ Kontakt mit den komplexen Abläufen im Bergbau zu treten und ein besseres Verständnis dafür zu erhalten, bei gleichzeitig minimalem Aufwand.

\section{Einsatz von VR und AR in der Lehre}

\subsection{Stand der Technik}

Der Einsatz von VR-Inhalten als Ergänzung zum traditionellen Lehrbetrieb hat in den letzten Jahren in Lehrpläne einiger Universitäten Eingang gefunden. Im Rahmen des EU-Projektes "SIMS" (Sustainable and Intelligent Mining Systems) wird beispielsweise ein vollständiges Grubengebäude als dreidimensionales Modell erstellt, in dem alle erdenklichen Situationen studiert werden können. Gestützt durch leistungsfähige Rechner, können Studenten der Luleå University of Technology verschiedene Szenarien durchspielen und Abläufe simulieren [1]. An der University of New South Wales steht den Studierenden ein voll ausgestattetes VR Kino zur Verfügung, in dem sie sich in einer virtuellen Bergbau-Umgebung bewegen können und dabei Maschinen und Ereignisse wie z. B. einen Grubenbrand, Wassereinbrüche und andere Szenarien erleben. Auf Wandhohe Leinwände werden dabei $360 \mathrm{Grad}$ 3D Bilder projiziert, die ein realitätsnahes Erleben der Bedingungen untertage oder im Tagebau ermöglichen. In der sicheren Umgebung einer 3D Simulation können so potentielle Gefahrensituationen und notwendige Rettungsmaßnahmen sicher trainiert werden, indem die Grenzen der traditionellen Lehre überschritten werden, hin zu experimentellem Lernen. [2]. Nennenswerte Bestrebungen zur Digitalisierung der Lehre finden sich auch an der RWTH Aachen: Im Rahmen des Programms "Fellowships für Innovationen in der Hochschullehre" wird an der Entwicklung und Erprobung digital gestützter Lehr- und Prüfungsformate gearbeitet (beispielsweise MOOCS, flipped/inverted classroom, Lernspiele, Simulationen, e-Prüfungen), um eine Neugestaltung von Modulen und Studienabschnitten unter konsequenter Nutzung digitaler Technologien zu schaffen.

Die oben geschilderten Bestrebungen bauen alle auf Hochleistungs-Rechnern auf, die jedoch einen großen Nachteil aufweisen: sie stehen nur lokal gebunden zur Verfügung. "MiReBooks" zielt auf Mobilität ab: Die digitalen Inhalte sind mittels VR-Brille und Smartphone erlebbar und damit unabhängig von einer bestimmten Lokalität. Diese mobile Lösung bietet erhebliche Vorteile, da sie überall einsetzbar und mit geringen Investitionskosten verbunden ist.

\subsection{Didaktisches Konzept}

Als Projektpartner in MiReBooks arbeitet die RWTH Aachen an der Erstellung eines didaktischen Konzeptes unter Berücksichtigung moderner Lehrmethoden. Ein Storyboard gibt die Struktur der Vorlesung vor, ähnlich einer Regieanweisung in einem Theaterstück. Anhand dieser Struktur kann der Lehrende die für den Inhalt der Vorlesung passenden Inhalte auswählen und ihren Einsatz im Hörsaal steuern. Den Studierenden stehen die Inhalte aus mehreren Quellen zur Verfügung, die entsprechend abwechselnd eingesetzt werden, z. B. Präsentationen, eBooks, Smartphones für die Anzeige von AR-Inhalten und VR-Brillen.

\subsection{Lehrmaterial}

Das Institut für Bergbaukunde der Montanuniversität Leoben arbeitet momentan an einer Versuchsvorlesung zum Thema "Gewinnungssprengung im Hartgesteins-Tagebau“. Dazu wurden im Dezember 2018 auf dem Steirischen Erzberg in Zusammenarbeit mit der TU Graz und der VA Erzberg mehrere Arbeitsschritte, darunter "Sprengen”, "Laden“ und „Fördern“ mit 360 Grad-Videokameras aufgenommen. (Abb. 2 und 3 ).

Am Institut für Maschinelles Sehen und Darstellen (ICG) der TU Graz werden die Rohdaten in VR-Filmdateien verarbeitet. Das ICG ist gleichzeitig der technische Hauptansprechpartner im Projekt MiReBooks. In Graz werden alle VR- und AR-Inhalte erzeugt, bzw. die Erstellung aller erforderlichen Medien koordiniert.

\subsection{Test-Vorlesungen}

In einer ersten Phase des Projektes werden zwei Test-Vorlesungen erstellt, die dazu dienen, die Methodik zu prüfen und Feedback von den Lehrenden und den Studierenden einzuholen, welche Medien für welche Lehrinhalte am besten eingesetzt werden können, und ob und wie Studierende und Lehrende mit VR- und AR-Elementen eine vertiefte Lernerfahrung erleben. Diese Test-Vorlesungen werden 2019 an den beteiligten Universitäten durchgeführt, um ein möglichst breites Feedback zu erhalten. Die Erkenntnisse, die in diesen Testvorlesungen gewonnen werden, fließen

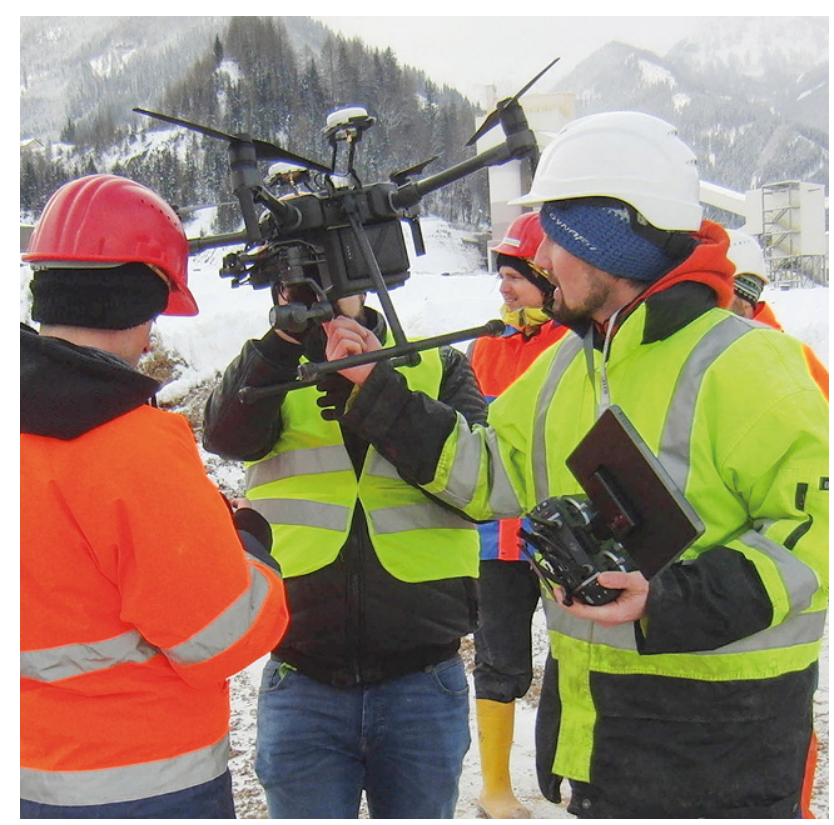

Abb. 2: Kameradrohne für Filmaufnahmen am Steirischen Erzberg 


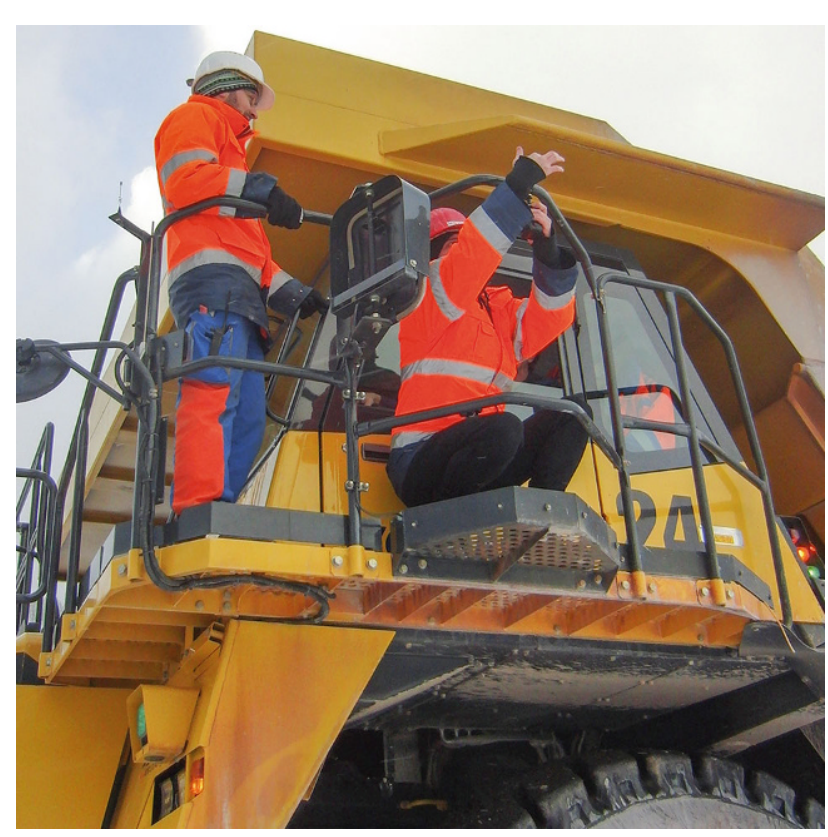

Abb. 3: Montage einer 3D Kamera an einem SLKW, Steirischer Erzberg

in die Gestaltung einer vollständigen Ausbildungsschiene für zukünftige Rohstoffingenieure ein. AR- und VR-Inhalte werden dabei eine wesentliche Rolle spielen.

\section{Projektkonsortium}

Die Produktion solcher Lehrmaterialien ist naturgemäß kostenintensiv und daher für einzelne Institutionen bzw. Universitäten alleine kaum zu bewältigen. Durch den Zusammenschluss mehrerer Partner zu einem Konsortium können alle individuellen Projekte in einem gemeinsamen Rahmen vereinigt werden. Diese Vorgangsweise erhöht nicht nur die Qualität der Lehrmaterialien, sondern verringert auch gleichzeitig den für die Erstellung erforderlichen Zeitaufwand. Die Partner dieses Konsortiums ergänzen sich mit ihren Kompetenzbereichen, wobei darauf geachtet wurde, andere Forschungsbereiche wie zum Beispiel Medizin, zu inkludieren, um von deren Erfahrung mit VR und $A R$ zu profitieren.

\subsection{Montanuniversität Leoben}

An der Montanuniversität Leoben ist das das gesamte Projektmanagement im Resources Innovation Center Leoben angesiedelt. Die Fachbeiträge, schwerpunktmäßig im Bereich Gebirgsmechanik und Tagebautechnik, steuert der Lehrstuhl für Bergbaukunde, Bergtechnik und Bergwirtschaft bei. Zudem stellt das Rektorat der Montanuniversität Leoben umfangreiche finanzielle Mittel zur Entwicklung eines breiten generischen Ausbildungsansatzes zur Verfügung.

\subsection{Technische Universität Graz (TU Graz)}

Die TU Graz ist mit zwei Instituten am Projekt "MiReBooks" beteiligt: dem Institut für Lehr- und Lerntechnologien und dem Institut für Maschinelles Sehen und Darstellen (ICG). Das Institut für Maschinelles Sehen und Darstellen (ICG) ist als federführender technischer Projektpartner für die Konzeption der Hardware zuständig.

\subsection{Technische Universität Bergakademie Freiberg}

Die TU Bergakademie Freiberg beteiligt sich ebenfalls mit zwei Instituten: dem Institut für Bergbau und Spezialtiefbau und der Professur für Virtuelle Realität und Multimedia. Einen speziellen Schwerpunkt der Freiberger Ingenieure stellt der Bereich Virtual Reality dar, besonders Visualisierungslösungen in hoher Auflösung in immersiven Umgebungen.

\subsection{Technische Universität Luleå}

Die Technische Universität Luleå als Schwedens nördlichste Universität beteiligt sich mit vier Bereichen an MiReBooks: 3D/4D Geologische Modellierungen von Erzkörpern und Gebieten, VR/AR/XR Lehrinhalte des Forschungsvorhabens SIMS (Sustainable and Intelligent Mining Systems) des Lehrstuhls für Bergbaukunde und Gebirgsmechanik, Expertise in 3D Visualisierungen und Computerspielen der Forschungsgruppen Visual Art und Pervasive and Mobile Computing, und Business Consulting.

\subsection{Rheinisch-Westfälische Technische Hoch- schule Aachen (RWTH)}

Die RWTH bringt ihre reichen Erfahrungen in der Methodik und Didaktik ins Projekt ein, die in den Bereichen Informationsmanagement im Maschinenbau (IMA) und Lern- und Wissensmanagement (ZLW) vorhanden sind. VR- und ARTechnologien spielen dabei eine wesentliche Rolle.

\subsection{Tallinn University of Technology (TTU)}

Die TTU ist ein aktiver Partner im Europäischen universitären Geschehen und bringt Vorlesungsinhalte für das Thema Hartgesteins-Tagebau ein, und erstellte auch eine der ersten Testvorlesungen.

\subsection{Universität Trient}

Das Institut für Industrielles Design der Universität Trient hat weitreichende Erfahrungen im Bereich VR- und AR-Unterstützung für medizinische und industrielle Anwendungen. 


\subsection{VTT Technical Research Centre of Finland Ltd (VTT)}

VTT ist ein international führendes Forschungsinstitut auf dem Gebiet der Augmented Reality, beheimatet in Espoo und Tampere. VTT's Forschungsgebiete liegen in den Bereichen Maschinelle Wartung, Architektur, Engineering und Bauindustrie.

\subsection{Epiroc Rock Drills AB}

Epiroc als führender Bergbauzulieferer steuert Bergbaumaschinenmodelle bei. Zudem werden die digitalen Handbücher auf Basis der früheren "Atlas Copco Excavation Handbooks" in vielen Universitäten in der Lehre eingesetzt.

\subsection{KGHM Cuprum}

Das Forschungszentrum des polnischen Kupferkonzerns KGHM ist spezialisiert auf die Erstellung von 3D Modellen und VR-Anwendungen zur Visualisierung von BergbauProzessen.

\subsection{Luossavaara-Kiirunavaara AB (LKAB)}

LKAB bringt praktische Erfahrung in Bergbautechnologie und Abläufen in das Projekt ein, ebenso werden Bereiche des Bergwerks als Versuchsgelände zur Verfügung gestellt.

\subsection{University of New South Wales}

Die University of New South Wales hat langjährige Erfahrung mit dem Einsatz von VR in der Lehre und in Sicherheitstrainings und innovativen Ausbildungskonzepten.

\subsection{VA Erzberg GmbH}

Der Steirische Erzberg stellt als hoch moderner mitteleuropäischer Tagebaubetrieb seine Abbautechnik und das bergbauliche Betriebsgelände für VR-Aufnahmen zur Verfügung.

\section{Ausblick}

Durch europaweite Kooperation mit kompetenten Partnern aus Industrie und Universitäten setzt die Montanuniversität mit dem Projekt MiReBooks einen wichtigen Schritt für die zukünftige Vermittlung modernen Bergbauwissens und wird damit den Erfordernissen einer an die Lerngewohnheiten der jungen Generation angepassten Art der Wissensvermittlung gerecht. Neue Vorlesungskonzepte und Inhalte werden in Form einer Serie von Lehrbüchern bzw. eBooks umgesetzt. MiReBooks wird es ermöglichen, dass Lernende ihr Studium oder ihre Berufsausbildung mit einem vertieften Verständnis ihrer Disziplin abschließen und sie optimal auf den digitalen Wandel im Bergbau vorbereiten. Darüber hinaus soll der jungen Generation ein zeitgemäßes modernes Bild der "High Tech Industrie Bergbau“ vermittelt werden. Daher ist auch angedacht, die zukünftigen VRund AR-basierten Bergbauhandbücher sowohl auf Ebene höherer technischer Bildungslehranstalten als auch auf betrieblicher Bergbauebene zugänglich zu machen.

MiReBooks verfolgt keinen „Distance Learning Ansatz", sondern der Unterricht wird nach wie vor an der Universität in der Interaktion von Lehrenden und Lernenden stattfinden.

Der Projektabschluss ist für das Jahr 2021 geplant, dann wird die universitäre Ausbildung Europa-übergreifend auf einen neuen Lehr-Standard gehoben.

Funding. Open access funding provided by Montanuniversität Leoben.

Open Access Dieser Artikel wird unter der Creative Commons Namensnennung 4.0 International Lizenz (http://creativecommons.org/licenses/ by/4.0/deed.de) veröffentlicht, welche die Nutzung, Vervielfältigung, Bearbeitung, Verbreitung und Wiedergabe in jeglichem Medium und Format erlaubt, sofern Sie den/die ursprünglichen Autor(en) und die Quelle ordnungsgemäß nennen, einen Link zur Creative Commons Lizenz beifügen und angeben, ob Änderungen vorgenommen wurden.

Hinweis des Verlags. Der Verlag bleibt in Hinblick auf geografische Zuordnungen und Gebietsbezeichnungen in veröffentlichten Karten und Institutsadressen neutral.

\section{Literatur}

1. SIMS Project: Sustainable Intelligent Mining System, https://www. simsmining.eu/ (24.01.2019)

2. UNSW Sydney, Australia, Faculty of Engineering: Virtual Reality Simulator, https://www.engineering.unsw.edu.au/mineralsenergy-resources/research/research-groups/innovative-learningand-teaching/virtual-reality-simulator-a-world-first (24.01.2019) 